\title{
LA-UR-00 -4942
}

Approved for public release; distribution is unlimited.

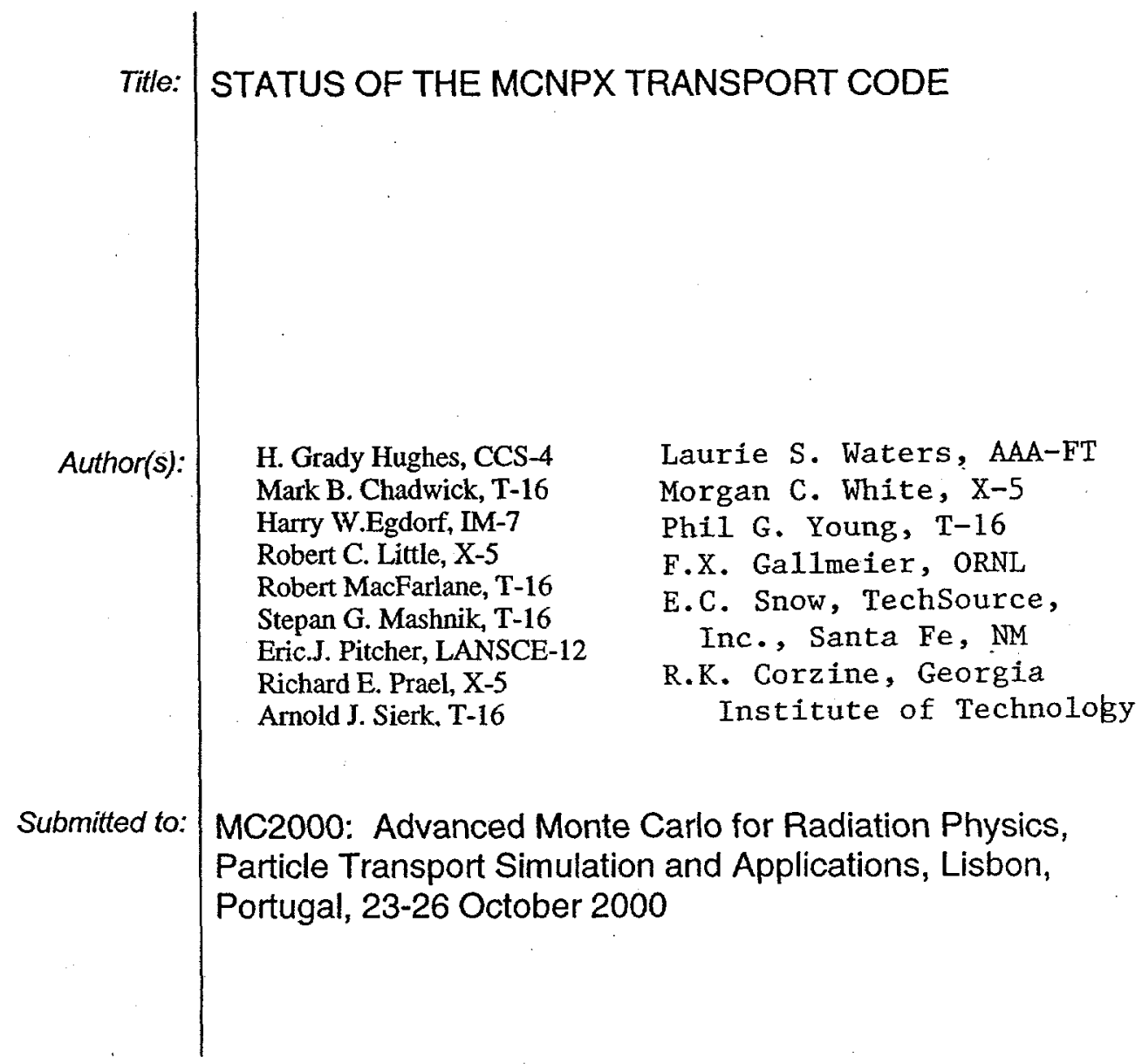

\section{Los Alamos NATIONAL LABORATORY}

Los Alamos National Laboratory, an affirmative action/equal opportunity employer, is operated by the University of Califomia for the U.S. Department of Energy under contract W-7405-ENG-36. By acceptance of this article, the publisher recognizes that the U.S. Government retains a nonexclusive, royalty-free license to publish or reproduce the published form of this contribution, or to allow others to do so, for U.S. Government purposes. Los Alamos National Laboratory requests that the publisher identify this article as work performed under the auspices of the U.S. Department of Energy. Los Alamos National Laboratory strongly supports academic freedom and a researcher's right to publish; as an institution, however, the Laboratory does not endorse the viewpoint of a publication or guarantee its technical correctness. 


\section{DISCLAIMER}

This report was prepared as an account of work sponsored by an agency of the United States Government. Neither the United States Government nor any agency thereof, nor any of their employees, make any warranty, express or implied, or assumes any legal liability or responsibility for the accuracy, completeness, or usefulness of any information, apparatus, product, or process disclosed, or represents that its use would not infringe privately owned rights. Reference herein to any specific commercial product, process, or service by trade name, trademark, manufacturer, or otherwise does not necessarily constitute or imply its endorsement, recommendation, or favoring by the United States Government or any agency thereof. The views and opinions of authors expressed herein do not necessarily state or reflect those of the United States Government or any agency thereof. 


\section{DISCLAIMER}

Portions of this document may be illegible in electronic image products. Images are produced from the best available original document. 


\section{Status of the MCNPX Transport Code}

H. G. Hughes ${ }^{1}$, M. B. Chadwick ${ }^{1}$, R. K. Corzine ${ }^{2}$, H. W. Egdorf ${ }^{1}$, F. X. Gallmeier $^{3}$, R. C. Little ${ }^{1}$, R. E. MacFarlane ${ }^{1}$, S. G. Mashnik ${ }^{1}$, E. J. Pitcher ${ }^{1}$, R. E. Prael ${ }^{1}$, A. J. Sierk ${ }^{1}$, E. C. Snow ${ }^{4}$, L. S. Waters ${ }^{1}$, M. C. White ${ }^{1}$, and P. G. Young ${ }^{1}$

1 Los Alamos National Laboratory, Los Alamos NM 87545 USA

${ }^{2}$ Georgia Institute of Technology, Atlanta GA 30332-0405 USA

3 Oak Ridge National Laboratory, Oak Ridge TN 37831 USA

4 TechSource, Inc., Santa Fe NM 87594-1057 USA

\section{Introduction}

The Monte Carlo particle transport code MCNPX and its associated data have been the focus of a major development effort at Los Alamos for several years [1]. The system has reached a mature state, and has become a significant tool for many intermediate- and high-energy particle transport applications. A recent version has been released to the Radiation Safety Information Computational Center (RSICC). A recent report [2] provides an overview of the code and an extensive set of references for the component physics modules used in the code.

In this paper we review the status of the developmental version of MCNPX, and describe some important new enhancements, including the use of evaluated nuclear data files for proton transport; the use of photonuclear reaction data; improved elastic and inelastic reaction cross sections for nucleons, antinucleons, pions, and kaons; and two new modes of operation of the code. We also illustrate the use of the new proton and photonuclear data in two representative applications.

\section{Table-Based Proton Transport}

Table-based charged-particle transport is a new feature of MCNPX and has been implemented for protons in the developmental version. Proton evaluations for energies up to $150 \mathrm{MeV}$ have been completed for 42 isotopes [3]. All evaluations include production cross sections for light particles, gamma rays, and heavy recoil particles, energy-angle correlated spectra for secondary light particles, and energy spectra for gamma rays and heavy recoil nuclei. The NJOY system [4] is used to process evaluated proton data into a tabular form appropriate for MCNPX. The MCNPX implementation uses tabular proton data to model nuclear reactions and large-angle scattering; continuous slowing down and multiple scattering models traditionally used in MCNPX for proton transport are used to model small-angle scattering.

Initial benchmarking of table-based MCNPX proton transport $[5,6]$ concentrated on neutron production from thick targets. MCNPX calculations, both 
with tables and with physics models, were compared to experiment for $30-\mathrm{MeV}$ protons on $\mathrm{Fe}, 68-\mathrm{MeV}$ protons on $\mathrm{C}$ and $\mathrm{Al}$, and $113-\mathrm{MeV}$ protons on $\mathrm{C}, \mathrm{Al}$, $\mathrm{Fe}$, and $\mathrm{Pb}$.

We have recently performed similar studies for a thin proton target. We have modeled the 43-MeV proton source from JAERI's TIARA AVF cyclotron. Protons impinge on a 3.6-mm thick ${ }^{7} \mathrm{Li}$ target. Resulting neutrons are constrained by an iron collimator $10.9 \mathrm{~cm}$ in diameter and $225 \mathrm{~cm}$ long. We have modeled this target, assuming a monoenergetic point proton source. The neutron flux is tallied on the surface exiting the collimator. In a report of the experimental results [7], the neutron flux is normalized to unity in an energy band between 36.3 and $45.5 \mathrm{MeV}$. We have normalized our calculated results in the same manner. Two MCNPX calculations were performed, one using proton tables and the other using the Bertini intranuclear cascade model in conjunction with a pre-equilibrium model.

Results for the experiment and the two calculations are shown in Fig. 1. Overall, neither calculation is in completely satisfactory agreement with the experiment. However, we observe that the width of the neutron peak more closely matches the experiment when proton tables are used. The Bertini model predicts a neutron peak that is lower in energy and much broader than observed.

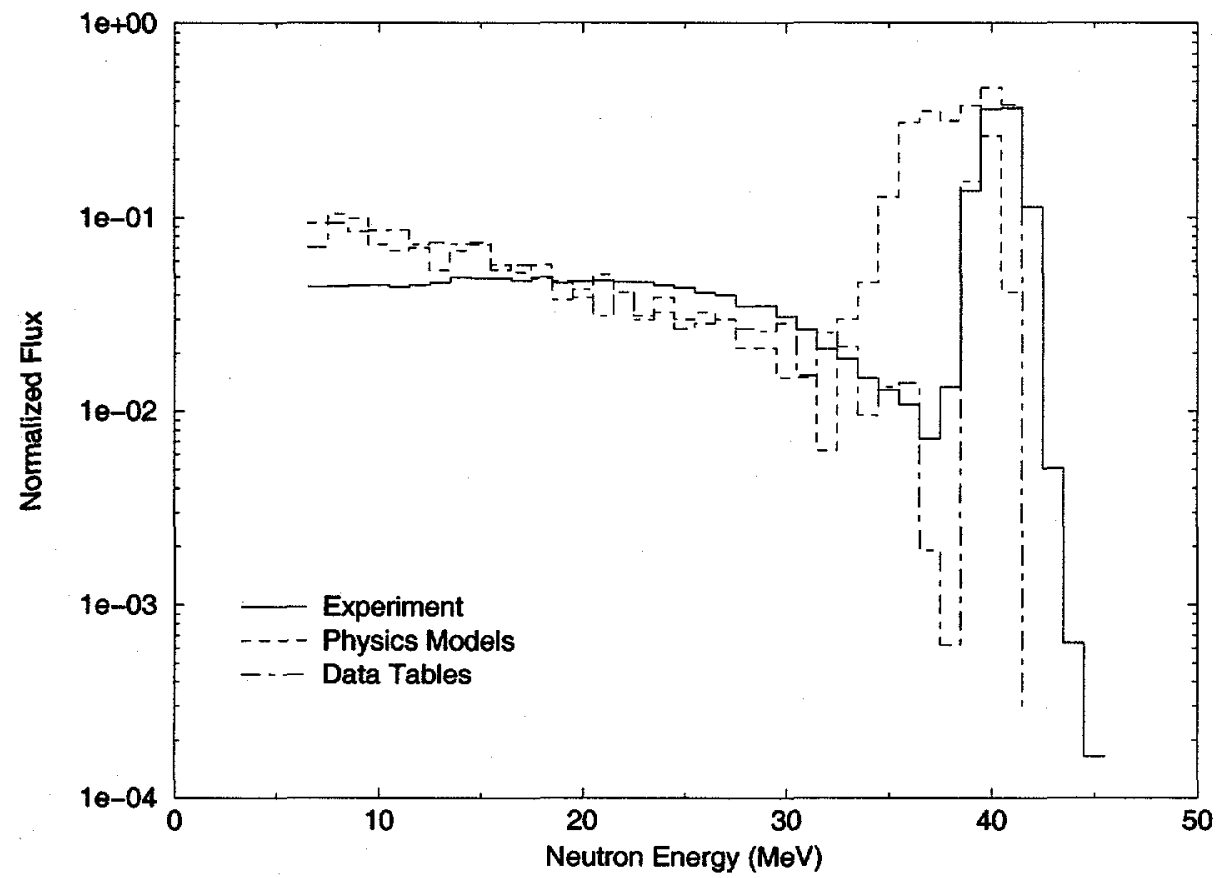

Fig. 1. Neutron Spectrum from 43-MeV Protons on ${ }^{7} \mathrm{Li}$. 


\section{Photonuclear Interactions}

Evaluated photonuclear data have recently become available for the first time [8]. These data include the doubly-differential cross sections necessary for Monte Carlo transport. MCNPX has been extended to use the newly available tabular data. Specifically, the distance to the next photon collision reflects the possibility of a photonuclear collision, and such collisions will produce a combination of neutrons, photons or light ions $(A \leq 4)$ for further transport. Thus, photonuclear events are fully integrated within a simulation. The implementation has been subjected to verification and validation testing [9]. Related work is in progress to enable the use of the Cascade-Exciton Model $[10,11]$ physics module to handle photonuclear events outside the tabular region.

The need for photonuclear physics in a Monte Carlo n-particle transport code has been generally accepted for some time. An interesting example of this need was the design of an electron beam stop for the Dual Axis Radiographic Hydrotest Facility (DARHT) at Los Alamos National Laboratory $[12,13]$. DARHT produces a $20-\mathrm{MeV}$ electron beam for use in photon radiography. The beamstop is designed to allow pulsing the accelerator while personnel are in the experimental area. The original design was a $20 \mathrm{~cm}$ diameter cylindrical plug with $7 \mathrm{~cm}$ of graphite backed by $25 \mathrm{~cm}$ of tungsten. The length of the tungsten backing was optimized to reduce the photon dose in the experimental area to acceptable levels. However, as seen in Fig. 2, this ignored a significant contribution to the

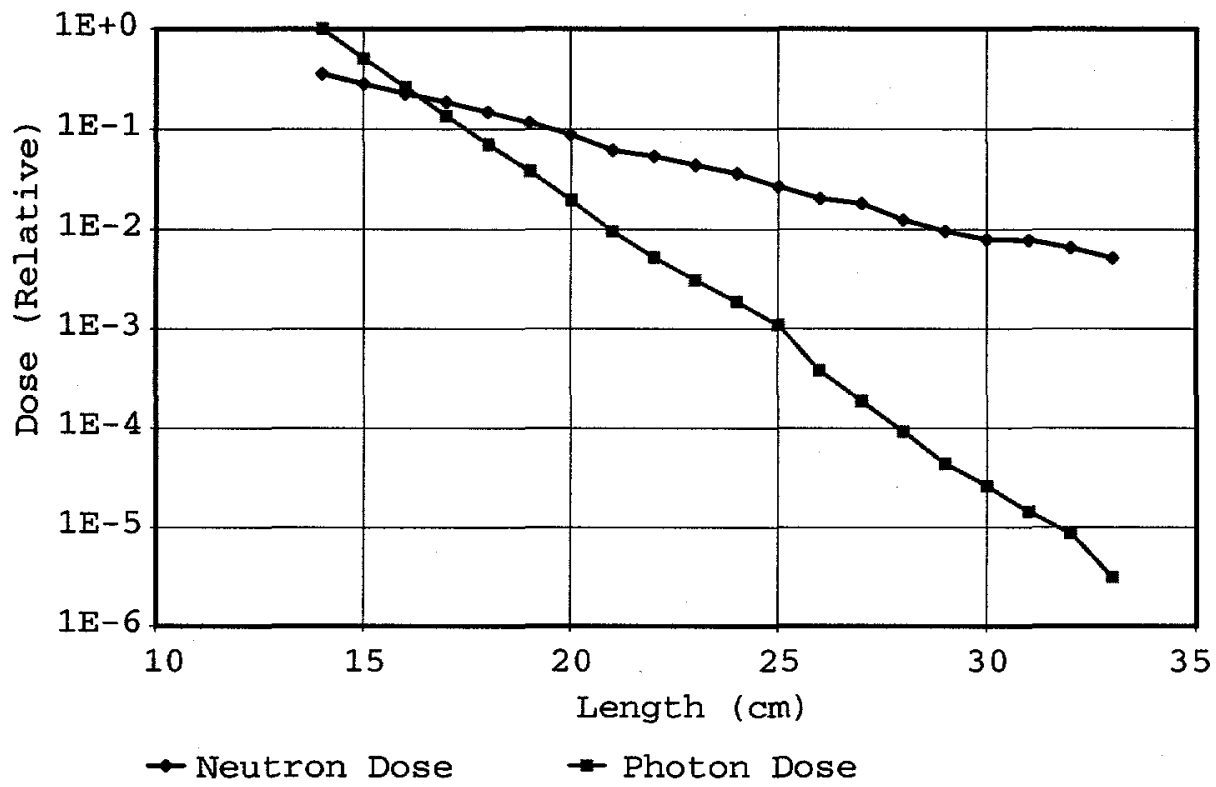

Fig. 2. Relative dose contribution from neutrons (diamonds) and photons (squares) for increasing thickness of tungsten in an electron beam stop. 
dose from neutrons. Further calculations were performed using a multi-step approach to estimate the neutron contribution. Based on these calculations, the tungsten in the beam stop was extended to bring the dose from neutrons to an acceptable level. Fig. 2 illustrates the new capability of MCNPX to calculate the contribution to the dose from neutrons and photons in a single simulation.

\section{Defined Elastic and Reaction Cross Sections}

Previously the concept of a reaction cross section for use with the intranuclear cascade model has been implicit in the model and not explicitly defined for use in the transport process. The new cross-section treatment [14] provides a defined (explicit) reaction cross section as well as a defined nuclear elastic cross section (previously utilized) in the absence of data libraries; these defined cross sections determine the transport process and constrain the corresponding reaction rates.

The new cross-section treatment has been implemented including an interpolation table for neutron elastic and reaction cross sections derived from the new 150-MeV MCNPX neutron libraries [3] (and some older 100-MeV libraries). Elastic scattering for protons is as implemented in LAHET2.8 [15]. Proton reaction cross sections are obtained by the methods of Barashenkov and Polanski [16], with Madland's optical model calculations [17] used where available, augmented by the coding of Tripathi $[18,19]$ below $1 \mathrm{GeV}$ and by the methods from FLUKA89 (Moehring formulation [20]) above $1 \mathrm{GeV}$. Beyond the range of the new tabular data, neutron reaction cross sections are similarly obtained. Elastic and reaction cross sections for pions are derived from the methods of Barashenkov and Polanski and of FLUKA89. For antinucleons and kaons, there are no elastic cross sections available, and the reaction cross sections are obtained only from the FLUKA89 methods.

\section{Other Enhancements}

With the implementation of the defined elastic and reaction cross sections in MCNPX, it becomes possible to treat primary beam transport [21] without nonelastic interactions or secondary particle production, determining attenuation by weight reduction using the prescribed reaction cross sections. This provides a relatively efficient method for examining small-angle dispersion of the primary beam and for testing methods for treating nuclear elastic scattering, multiple Coulomb scattering, and energy straggling. It also provides a necessary feature for the development of next-event estimators for higher energy neutron flux above the energies where standard MCNP methods, using evaluated data libraries, are commonly used.

A cross-section generation option has been adapted from LAHET [22] and implemented in MCNPX. Using this option, the interaction models are accessed directly for any source particle, and the interaction products are recorded to a history file. This history file may be subsequently processed by a postprocessing 
code (called XSEX3) to create double differential cross sections for any of the interaction models included in MCNPX.

Finally, the developmental version of MCNPX includes a new atomic mass data base [23] and the code to access it; in the current version it is used by all the physics packages shared by LAHET and MCNPX.

\section{References}

1. H. G. Hughes, K. J. Adams, M. B. Chadwick, J. C. Comly, S. C. Frankle, J. S. Hendricks, R. C. Little, R. E. Prael, L. S. Waters, P. G. Young: 'MCNPX - The LAHET/MCNP Code Merger'. In: Proceedings of the Third Workshop On Simulating Accelerator Radiation Environments (SARE3), May 7-9, 1997, Tsukuba, Japan (KEK Proceedings 97-5, June 1997) pp. 44-51.

2. H. G. Hughes, K. J. Adams, M. B. Chadwick, J. C. Comly, L. J. Cox, H. W. Egdorf, S. C. Frankle, J. S. Hendricks, R. C. Little, R. E. MacFarlane, S. G. Mashnik, R. E. Prael, A. J. Sierk, L. S. Waters, M. C. White, P. G. Young, F. X. Gallmeier, E. C. Snow: 'MCNPX for Neutron-Proton Transport'. In: Mathematics and Computation, Reactor Physics and Environmental Analysis in Nuclear Applications, September 27-30, 1999, Madrid, Spain, ed. by J. M. Aragones, C. Ahnert, and O. Cabellos (Senda Editorial, Spain, 1999) pp. 939-948.

3. M. B. Chadwick, P. G. Young, S. Chiba, S. Frankle, G. M. Hale, H. G. Hughes, A. J. Koning, R. C. Little, R. E. MacFarlane, R. E. Prael, L. S. Waters: 'Cross Section Evaluations to $150 \mathrm{MeV}$ for Accelerator-Driven Systems and Implementation in MCNPX'. Nucl. Sci. Eng. 131 (1999) pp. 293-328.

4. R. E. MacFarlane, D. W. Muir: 'The NJOY Nuclear Data Processing System, Version 91'. Los Alamos National Laboratory report LA-12740-M (1994).

5. M. B. Chadwick, H. G. Hughes, R. C. Little, E. J. Pitcher, P. G. Young: 'Nuclear Data for Accelerator-Driven Systems'. Los Alamos National Laboratory report LA-UR-00-30 (2000), to be published in Progress in Nuclear Energy.

6. M. B. Chadwick, H. G. Hughes, R. C. Little, E. J. Pitcher, P. G. Young: 'Physics Models and Nuclear Data Evaluations for Enhanced Monte-Carlo Transport'. Proceedings of this Conference.

7. N. Nakao, H. Nakashima, T. Nakamura, S. Tanaka, S. Tanaka, K. Shin, M. Baba, Y. Sakamoto, Y. Nakane: 'Transmission Through Shields of Quasi-Monoenergetic Neutrons Generated by $43-\mathrm{MeV}$ and 68-MeV Protons - I: Concrete Shielding Experiment and Calculation for Practical Application'. Nucl. Sci. Eng. 124 (1996) pp. 228-242.

8. P. Oblozinsky, M. B. Chadwick, A. I. Blokhin, T. Fukahori, Y.-O. Lee, M. N. Martins, V. V. Varlamov, B. Yu, Y. Han, S. F. Mughabghab, J. Zhang: Handbook on Photonuclear Data for Applications: Cross Sections and Spectra. International Atomic Energy Agency TEC-DOC In Press (To be published in 2000; see http://iaeand.iaea.org/photonuclear for draft).

9. M. C. White: 'Development and Implementation of Photonuclear Cross-Section Data for Mutually Coupled Neutron-Photon Transport Calculations in the Monte Carlo N-Particle (MCNP) Radiation Transport Code'. Los Alamos National Laboratory report LA-13744-T (July 2000).

10. S. G. Mashnik, A. J. Sierk, O. Bersillon, T. A. Gabriel: 'Cascade-Exciton Model Detailed Analysis of Proton Spallation at Energies From $10 \mathrm{MeV}$ to 5 GeV'. Nucl. 
Instr. Meth. A414 (1998) p. 68. (Los Alamos National Laboratory Report LAUR-97-2905)

11. F. X. Gallmeier: 'Implementation of the CEM-Code into the MCNPX-Code'. In: Proceedings of the Fourth Workshop on Simulating Accelerator Radiation Environments (SARE 4), September 14-16, 1998, Knoxville, Tennessee, ed. by Tony A. Gabriel, Oak Ridge National Laboratory (1998) pp. 131-139.

12. T. H. Brown: 'Shielding Design of the Electron Beam Stop for the Dual-Axis Radiographic Hydrotest Facility (DARHT)'. In: Proceedings of the 1996 Topical Meeting on Radiation Protection and Shielding, April 21-25, 1996, No. Falmouth, Massachusetts (American Nuclear Society, 1996) pp. 102-109.

13. M. B. Chadwick, T. H. Brown, R. C. Little: 'Photoneutron Production in the Electron Beam Stop for the Dual Axis Radiographic Hydrotest Facility (DARHT)'. In: Proceedings of the ANS Radiation Protection and Shielding Topical Conference, April 19-23, 1998, Nashville, Tennessee (American Nuclear Society, 1998) Vol. II, pp. 356-363.

14. R. E. Prael, A. Ferrari, R. K. Tripathi, A. Polanski: 'Comparison of Nucleon Cross Section Parameterization Methods for Medium and High Energies'. In: Proceedings of the Fourth Workshop on Simulating Accelerator Radiation Environments (SARE4), September 14-16, 1998, Knoxville, Tennessee, ed. by Tony A Gabriel, Oak Ridge National Laboratory (1998) pp. 171-181.

15. R. E. Prael, D. G. Madland: 'A Nucleon-Nucleus Elastic Scattering Model for LAHET'. In: Proceedings of the 1996 Topical Meeting on Radiation Protection and Shielding, April 21-25, 1996, No. Falmouth, Massachusetts (American Nuclear Society, 1996) pp. 251-257.

16. V. S. Barashenkov, A. Polanski: 'Electronic Guide for Nuclear Cross Sections'. Comm. JINR E2-94-417, Dubna (1994).

17. D. G. Madland: 'Recent Results in the Development of a Global Medium-Energy Nucleon-Nucleus Optical-Model Potential,' In: Proceedings of a Specialist's Meeting on Preequilibrium Reactions, Semmering, Austria, February 10-12, 1988, ed. by Brigitte Strohmaier (OECD, Paris, 1988) pp. 103-116.

18. R. K. Tripathi, F. A. Cucinotta, J. W. Wilson: 'Universal Parameterization of Absorption Cross Sections'. NASA Technical Paper 3621 (January 1997).

19. R. K. Tripathi, J. W. Wilson, and F. A. Cucinotta: 'New Parameterization of Neutron Absorption Cross Sections'. NASA Technical Paper 3656 (June 1997).

20. H. J. Moehring: 'Hadron-nucleus Inelastic Cross-sections for Use in Hadroncascade Calculations at High Energies'. CERN report TIS-RP/116 (October 1983).

21. R. E. Prael: 'Primary Beam Transport Methods in LAHET'. In: Transactions of the 1999 Annual Meeting, June 6-10, 1999, Boston, Massachusetts (American Nuclear Society, 1999) pp. 325-326.

22. Richard E. Prael, Henry Lichtenstein: 'User Guide to LCS: The LAHET Code System'. Los Alamos National Laboratory report LA-UR-89-3014 (September 1989).

23. R. E. Prael: 'Upgrading Physics Packages for LAHET/MCNPX'. In: Proceedings of the 2nd International Topical Meeting on Nuclear Applications of Accelerator Technology, September 20-23, 1998, Gatlinburg, Tennessee (American Nuclear Society, 1998) pp. 276-280. 